031 INJURY SURVEILLANCE AT THE 17/U \& 19/U AUSTRALIAN NATIONAL NETBALL CHAMPIONSHIPS AND THE EFFECT OF A FOOT BLISTER PREVENTION INTERVENTION

\footnotetext{
1,2Erin Smyth, ${ }^{3}$ Laura Piromalli, ${ }^{3}$ Alanna Antcliff, ${ }^{1}$ Phillip Newman, ${ }^{1,2}$ Gordon Waddington, ${ }^{4} J u a n i t a$ Weissensteiner, ${ }^{2,5}$ Michael Drew. 'University of Canberra, Canberra, Australia; ${ }^{2}$ Australian Institute of Sport, Canberra, Australia; ${ }^{3}$ Netball Australia, Canberra, Australia; ${ }^{4}$ New South Wales Office of Sport, Sydney, Australia; ${ }^{5}$ Australian Collaboration for Research into Injury in Sport and its Prevention (ACRISP), Perth, Australia
}

\subsection{6/bjsports-2021-IOC.29}

Background Pre-elite athletes are at increased risk of injury/illness due to rapid increases in training load, insufficient rest and recovery, growth and a lack of support services compared to elite athletes.

Objective Surveillance of incidence, site, nature and mechanisms of injury/illness at the 2018 \& 2019 17/U \& 19/U Australian Netball National Championships (ANNC) and reduce injuries at the 2019 ANNCs.

Design Prospective observational cohort study.

Setting 2018 \& 2019 17/U \& 19/U ANNC's.

Patients (or Participants) One hundred and ninety-two pre-elite athletes were observed each year.

Interventions (or Assessment of Risk Factors) A foot blister prevention pack and advice regarding an injury prevention program were provided to each athlete 6 weeks prior to 2019 ANNC's. Main Outcome Measurements Injuries were recorded prospectively by team physiotherapists using medical attention data collection methods at the 2018 \& 2019 ANNCs.

Results The most frequently recorded medical attention injury diagnoses in 2018 were ankle sprain $(n=14,13.6 \%)$, foot blisters $(\mathrm{n}=11,10.7 \%)$, and lumbar pain $(\mathrm{n}=10,9.7 \%)$. In 2019 there were $16(16.8 \%)$ ankle sprains, followed by $12(12.6 \%)$ episodes of lumbar pain and 8 (8.4\%) foot blisters. In 2018 there were $22(21 \%)$ sports incapacity injuries with ankle sprain $(n=4,18.2 \%)$, anterior cruciate ligament (ACL) rupture $(n=3,13.6 \%)$ and concussion $(n=3,13.6 \%)$ recorded most frequently. In 2019, there were $12(12.6 \%)$ sports incapacity injuries with concussion $(n=5,5.3 \%)$ recorded most frequently followed by ACL rupture $(n=4,4.2 \%)$. The blister prevention intervention reduced the number of foot blisters at the 2019 ANNCs (IRR $0.730 .58-0.91, p=0.002$ ).

Conclusions This is the first publication in recent times to articulate tournament injury rates for pre-elite netball athletes. Ankle sprains are the highest medical attention injury in preelite netball athletes. This has not changed over the past 30 years indicating current injury prevention interventions for ankle sprain are not effective. Blister prevention packs and advice reduced the number of foot blisters.

\section{DOES MENTAL FATIGUE NEGATIVELY AFFECT FUNCTIONAL PERFORMANCE TESTS USED TO SCREEN FOR LOWER EXTREMITY INJURY RISK?}

\footnotetext{
${ }^{1}$ Jo Verschueren, ${ }^{1}$ Bruno Tassignon, 'Jeroen Van Cutsem, 'Bart Roelands, ${ }^{2}$ Evert Verhagen, ${ }^{1,3}$ Romain Meeusen. ${ }^{1}$ Human Physiology and Sports Physiotherapy Research Group, Faculty of Physical Education and Physiotherapy, Vrije Universiteit Brussel, Brussels, Belgium; ${ }^{2}$ Amsterdam Collaboration on Health and Safety in Sports, Department of Public and Occupational Health, Amsterdam Movement Sciences, Amsterdam UMC, Vrije Universiteit Amsterdam, Amsterdam, Netherlands; ${ }^{3}$ Strategic Research Program 'Exercise and the Brain in Health and Disease: the added value of Human-Centered Robotics', Vrije Universiteit Brussel, Brussels, Belgium
}

10.1136/bjsports-2021-IOC.30
Background A lot of controversy exists about how fatigue affects injury risk. Within the fatigue spectrum, mental fatigue impairs visuomotor skills, reaction time and decision-making. Recently, adaptability has been put forward as an important driver in understanding injury occurrence and injury prevention. It is hypothesised that mental fatigue decreases an athlete's adaptability by means of an increased visuomotor reaction time and diminished ability for decision-making and visuomotor skill execution.

Objective To assess the impact of mental fatigue on functional tests used in injury risk profiling and if mental fatigue decreases an athlete adaptability.

Design Randomized counterbalanced cross-over design.

Setting Primary prevention.

Participants Fourteen healthy participants (age $=22 \pm 1$ years; height $=176.9 \pm 8.4 \mathrm{~cm}$; weight $=69.7 \pm 10.4 \mathrm{~kg}$ ). Interventions Mental fatigue was induced by a 90-minute $100 \%$ incongruent Stroop color word test, while the control task included watching a 90-minute documentary.

Main Outcome Measurements Y-balance test (YBT), Reactive balance test (RBT), Single leg hop test (SLH) and countermovement jump (CMJ) performance was evaluated pre-post mental fatigue. Mental fatigue was evaluated using the M-VAS, Stroop and Eriksen-Flanker task performance outcomes.

Results Mental fatigue was successfully induced at the subjective level with a significant increase in M-VAS $(\mathrm{p}<0.001)$, with no decrease in performance on the Stroop and Eriksen-Flanker task. Mental fatigue did not affect YBT, SLH and the CMJ performance. However, RBT accuracy significantly decreased in mental fatigue condition $(p=0.024)$, while the visuomotor reaction time of the RBT remained unaffected.

Conclusions This is the first experiment to illustrate that mental fatigue affects functional adaptability, as accuracy in response to environmental stimuli decreases. However, traditional functional test perfmance remains unaffected. Therefore, both neurocognitive functional tests and mental fatigue responses should be considered within injury risk profiling strategies. Prospective study designs within the injury prevention domain should include neurocognitive functional tests to investigate the possible relationship of these tests with sports injuries.

\section{STRONG PREDICTIVE POWER OF MUSCLE FIBRE TYPOLOGY ON HAMSTRING STRAIN INJURY RISK IN PROFESSIONAL SOCCER}

${ }^{1}$ Eline Lievens, ${ }^{1}$ Kim Van Vossel, ${ }^{1}$ Freek Van de Casteele, ${ }^{2}$ Evi Wezenbeek, ${ }^{2}$ Erik Witvrouw, ${ }^{1}$ Wim Derave. 'Department of Movement and Sports Sciences, Ghent University, Ghent, Belgium; 'Department of Rehabilitation Sciences and Physiotherapy, Ghent University, Ghent, Belgium

\subsection{6/bjsports-2021-IOC.31}

Background Hamstring strain injuries (HSI) are the most prevalent injuries in team sports and occur more frequently in the fatigued phases of a match. In the search for interindividual factors determining increased muscle fatigue-and thus HSI risk-the muscle fibre type composition (fast-twitch vs slowtwitch muscle typology) is a likely candidate.

Objective The objective of this study was to identify whether the muscle fibre typology is a risk factor for HSI.

Design Prospective cohort study over 3 consecutive seasons 